\title{
Die Europäische Union in der Währungskrise: Eckdaten und Schlüsselentscheidungen
}

\author{
Tobias Kunstein und Wolfgang Wessels*
}

Auch wenn die europapolitische Berichterstattung die Europäische Union mit großer Regelmäßigkeit in einer Krise sieht, ist die derzeitige Notlage der gemeinsamen Währung von besonderer Dramatik. Tatsächlich stellt die andauernde Krise die Existenz der europäischen Wirtschafts- und Währungsunion (WWU) infrage und gefährdet damit auch wesentliche Fundamente der Europäischen Union: Die zum Teil in langen Nachtsitzungen getroffenen Entscheidungen der Staats- und Regierungschefs laufen auf eine umfassende Neugestaltung der WWU und ihres politischen Rahmens hinaus. Während über das Ende der Krise Ende 2011 nur spekuliert werden kann, ist davon auszugehen, dass Form und Intensität der Reaktionen auf diese Herausforderung gravierende Auswirkungen auf die Politikbereiche und die Institutionen der Europäischen Union, auf die Mitgliedstaaten der Eurozone (Eurostaaten), aber auch auf andere EU-Mitgliedstaaten haben werden.

\section{Die Krise im Überblick: zwei Phasen}

Grob lassen sich zwei Phasen der Krise unterscheiden. Bei der ersten Phase von Ende 2007 bis 2009 handelt es sich um eine Finanzkrise, die in eine Wirtschaftskrise mündet. Hoher Abschreibungsbedarf bei notleidenden Krediten bringt zahlreiche Finanzinstitute an den Rand des Zusammenbruchs. Der Vertrauensverlust der Finanzmarktakteure untereinander stellt dabei die Grundvoraussetzung des kreditbasierten globalen Wirtschaftssystems infrage. Diese erste Phase der Krise geht nahtlos und konsequenterweise über in eine zweite Phase, die sich als Staatsschuldenkrise charakterisieren lässt. Durch massive Stützungsprogramme für marode Banken und Bemühungen, den wirtschaftlichen Abschwung abzumildern, schießen ab 2009 die Defizite in den Staatshaushalten fast aller EU-Mitgliedstaaten in die Höhe. Für die Eurozone ergibt sich dabei eine besondere Verwundbarkeit: Die erhoffte wirtschaftliche Konvergenz ihrer Mitgliedstaaten nach der Einführung der Gemeinschaftswährung im Jahr 1999 hat nicht oder nicht in ausreichendem Maß stattgefunden. Darüber hinaus wurde das im Vertrag von Maastricht fixierte und um den sogenannten Stabilitäts- und Wachstumspakt ergänzte Regelwerk zur Vermeidung übermäßiger Haushaltsdefizite ${ }^{1}$ bereits in wirtschaftlich verhältnismäßig stabilen Zeiten - nicht zuletzt von Deutschland - verletzt und nachhaltig ausgehöhlt. ${ }^{2}$ Mit dem Ausbruch der Krise wird das Dilemma offensicht-

* Tobias Kunstein, Wissenschaftlicher Mitarbeiter, Forschungsinstitut für Politische Wissenschaft und Europäische Fragen, Universität zu Köln.

Prof. Dr. rer. pol. Wolfgang Wessels, Jean Monnet Lehrstuhl, Forschungsinstitut für Politische Wissenschaft und Europäische Fragen, Universität zu Köln.

Eine frühere Fassung dieses Artikels erschien als Vorwort für einen Nachdruck der 12. Auflage von Werner Weidenfeld/Wolfgang Wessels (Hrsg.): Europa von A-Z. Taschenbuch der Europäischen Integration, Baden-Baden 2011.

1 Art. 126 Vertrag über die Europäische Union (EUV); Protokoll (Nr. 12) über das Verfahren bei einem übermäBigen Defizit, in: Amtsblatt der EU, Nr. C 83 vom 30. März 2011, S. 279-280. Der 1997 geschaffene Stabilitäts- und Wachstumspakt, der aus einer Entschließung des Europäischen Rates sowie zwei - im Jahr 2005 überarbeiteten - Verordnungen des Ministerrats besteht, präzisiert die vertraglichen Vorgaben.

2 Als Überblick zu den ersten zehn Jahren Eurozone vgl. Daniela Schwarzer: Zehn Jahre Governance der Eurozone: ökonomische Bilanz und institutionelle Dynamiken jenseits der Vertragsrevisionen, in: integration 1/ 2009, S. 17-32. 
lich. Einerseits ist den weniger wettbewerbsfähigen Staaten als Mitglieder der Eurozone der verhältnismäßig , leichte“ Weg zur Anpassung, eine Abwertung der eigenen Währung, versperrt. Andererseits hatte bislang Einigkeit bestanden, dass die Gemeinschaft der Eurostaaten nicht für Schulden einzelner Staaten haftet, um Anreize zum Schuldenmachen auf Kosten der Allgemeinheit zu vermeiden. ${ }^{3}$ Angesichts des Ausmaßes der Krise zeichnet sich jedoch ab, dass die Defizite in den Staatshaushalten einiger Eurostaaten mit den verbleibenden Instrumenten der nationalen Wirtschaftspolitik nicht mehr tragbar sind. Jede beliebige Kombination von Sparanstrengungen, wachstumsfördernden Maßnahmen und Steuererhöhungen erscheint selbst zur kurzfristigen Deckung der Finanzierungslücke vergeblich. Die wirtschaftlich stabileren Mitglieder der Eurozone finden sich in einer nur wenig komfortableren Position: Können sie angesichts der unkalkulierbaren Konsequenzen für ihre eigenen Volkswirtschaften und Bankensysteme das Risiko eines Staatsbankrotts innerhalb der eng verflochtenen Währungsunion eingehen? Können sie Szenarien ignorieren, bei denen sich lokale Feuer zu einem Flächenbrand ausweiten?

\section{Tabelle 1: Chronologie der Währungskrise: Eckpunkte und Schlüsselentscheidungen}

\begin{tabular}{|c|c|}
\hline Ab August 2007 & $\begin{array}{l}\text { Ungleichgewichte in einigen Finanzmarktsegmenten. Unterstützungsmaßnah- } \\
\text { men der EZB. Nationale Regierungen legen Bankenrettungsprogramme auf. }\end{array}$ \\
\hline 15. September 2008 & US-Bank Lehman Brothers muss Insolvenz anmelden. \\
\hline Oktober 2008 & EZB trifft zusätzliche temporäre Krisenmaßnahmen. \\
\hline 12. Oktober 2008 & $\begin{array}{l}\text { Erstes Treffen der Staats- und Regierungschefs der Eurozone (Euro-Gipfel) in } \\
\text { Paris. }\end{array}$ \\
\hline 15.-16. Oktober 2008 & Europäischer Rat bestätigt Willen zu konzertiertem Vorgehen. \\
\hline 20. Oktober 2009 & $\begin{array}{l}\text { Neugewählte griechische Regierung veröffentlicht drastisch korrigierte Defi- } \\
\text { zitzahlen. }\end{array}$ \\
\hline 11. Februar 2010 & Erster informeller EU-Krisengipfel zu Griechenland. \\
\hline 25.-26. März 2010 & $\begin{array}{l}\text { Europäischer Rat und Euro-Gipfel in Brüssel. Die Mitglieder der Eurozone } \\
\text { verpflichten sich zu koordinierten bilateralen Darlehen, falls sich Griechenland } \\
\text { an den Finanzmärkten nicht mehr refinanzieren kann. Der Europäische Rat } \\
\text { richtet Van Rompuy Task Force ein. }\end{array}$ \\
\hline 23. April 2010 & $\begin{array}{l}\text { Griechenland ruft die in Aussicht gestellten Hilfen ab und verpflichtet sich zu } \\
\text { einem harten Reform- und Sparkurs, der durch Kommission, EZB und IWF } \\
\text { überwacht wird. }\end{array}$ \\
\hline 7.-8. Mai 2010 & $\begin{array}{l}\text { Staats- und Regierungschefs der Eurozone in Brüssel verabschieden einen } \\
\text { „Rettungsschirm für den Euro“. }\end{array}$ \\
\hline 9.-10. Mai 2010 & $\begin{array}{l}\text { EU-Finanzminister einigen sich in einer außerordentlichen Ratssitzung auf die } \\
\text { Details des Rettungsschirms, später bezeichnet als „European Financial Stability } \\
\text { Facility“ (EFSF) und „European Financial Stabilisation Mechanism“ (EFSM). }\end{array}$ \\
\hline 10. Mai 2010 & EZB beginnt, Staatsanleihen der Krisenstaaten zu kaufen. \\
\hline 7. September 2010 & $\begin{array}{l}\text { Ministerrat beschließt einen als „Europäisches Semester“ bezeichneten Zyklus } \\
\text { zur Koordinierung der Wirtschaftspolitik. }\end{array}$ \\
\hline
\end{tabular}

3 Zur Auslegung des Art. 125 EUV als ein generelles Verbot siehe Martin Seidel: Aktuelle Probleme der europäischen Währungsunion, in: integration 4/2010, S. 334-349, hier S. 344-346. 


\begin{tabular}{|c|c|}
\hline 29. September 2010 & $\begin{array}{l}\text { Europäische Kommission stellt Paket von sechs Gesetzgebungsvorschlägen im } \\
\text { Bereich des wirtschaftspolitischen Regierens vor (Sixpack). }\end{array}$ \\
\hline 18. Oktober 2010 & $\begin{array}{l}\text { Angela Merkel and Nicolas Sarkozy fordern nach einem Treffen in Deauville } \\
\text { eine Vertragsänderung, die einen permanenten Mechanismus zur Rettung in- } \\
\text { solventer Staaten erlauben soll. }\end{array}$ \\
\hline 21. Oktober 2010 & Van Rompuy Task Force legt ihren Bericht für den Europäischen Rat vor. \\
\hline 28. November 2010 & Irland erhält als erstes Land einen Notkredit des Rettungsschirms. \\
\hline 16.-17. Dezember 2010 & $\begin{array}{l}\text { Europäischer Rat beschließt eine Ergänzung der EU-Verträge zur Einrichtung } \\
\text { eines permanenten „European Stability Mechanism“(ESM). }\end{array}$ \\
\hline 11. März 2011 & $\begin{array}{l}\text { Euro-Gipfel im Rahmen einer außerordentlichen Sitzung des Europäischen Ra- } \\
\text { tes zur Situation in Libyen. Absprache zur Erhöhung der Kapazität der EFSF } \\
\text { und Einrichtung eines „Paktes für den Euro“, später als „Euro-Plus-Pakt“ be- } \\
\text { zeichnet. }\end{array}$ \\
\hline 24.-25. März 2011 & $\begin{array}{l}\text { Europäischer Rat verabschiedet auf Basis der Beschlüsse vom 11. März } 2011 \\
\text { ein ,umfassendes“ Maßnahmenpaket zur Bekämpfung der Krise und nimmt die } \\
\text { Vertragsänderung zur Einrichtung des ESM an. }\end{array}$ \\
\hline 17. Mai 2011 & Portugal erhält einen Notkredit des Rettungsschirms. \\
\hline 21. Juli 2011 & $\begin{array}{l}\text { Die } 17 \text { Staats- und Regierungschefs der Eurozone verabschieden ein zweites } \\
\text { Hilfspaket für Griechenland und erweitern die Kompetenzen der EFSF. }\end{array}$ \\
\hline $\begin{array}{l}\text { 28. September und } \\
\text { 4. Oktober } 2011\end{array}$ & $\begin{array}{l}\text { Europäisches Parlament (28. September 2011) und Ministerrat (4. Oktober } \\
\text { 2011) verabschieden das sogenannte Sixpack. }\end{array}$ \\
\hline 23. Oktober 2011 & $\begin{array}{l}\text { Doppelgipfel der Staats- und Regierungschefs der Europäischen Union und der } \\
\text { Eurozone. Die eigentliche Beschlussfassung wird auf einen weiteren Gipfel am } \\
\text { 26. Oktober } 2011 \text { vertagt. }\end{array}$ \\
\hline 26. Oktober 2011 & Euro-Gipfel beschließt Maßnahmen zur Stabilisierung der Eurozone. \\
\hline 8.-9. Dezember 2011 & $\begin{array}{l}\text { Euro-Gipfel und Europäischer Rat verabschieden weitere Maßnahmen hin zu } \\
\text { einer ,fiskalpolitischen Stabilitätsunion“, die stärker als bisher in nationale } \\
\text { Haushalte eingreift. Ein Teil dieser Regeln soll primärrechtlich verankert wer- } \\
\text { den. Allein Großbritannien sperrt sich gegen eine entsprechende Änderung der } \\
\text { EU-Verträge. Die übrigen } 26 \text { Staaten streben daher eine zwischenstaatliche } \\
\text { Vereinbarung außerhalb der bestehenden Verträge an. }\end{array}$ \\
\hline
\end{tabular}

Quelle: Eigene Darstellung.

Auch wenn die politischen Führungen in der Europäischen Union und den Mitgliedstaaten diese Frage mit einem immer wieder betonten ,Nein “ beantworten, besteht nach wie vor Uneinigkeit über den Weg zur Lösung der Krise - mit welchen Mitteln soll das Feuer gelöscht werden? Nicht zuletzt weil der Puls der Finanzmärkte einen Zeitdruck erzeugt, ${ }^{4}$ der mit den gewohnten Abläufen des Aushandelns von europäischen Kompromissen über mehrere Handlungsebenen und Institutionen hinweg nur schwer kompatibel ist, ist das Resultat eine lange Reihe von Gipfeln, Sondergipfeln und Rettungspaketen, die häufig nur eine aufschiebende Wirkung zeigen (siehe Tabelle 1). Besonderes Interesse weckt dabei, wie Europäischer Rat (die Institution der Staats- und Regierungschefs der 27 EU-Mitgliedstaaten sowie der Präsidenten des Europäischen Rates und der Kommission) und Euro-Gipfel (die

4 Zum Einfluss von Rating-Agenturen und Finanzmarktregeln, insbesondere zum Risikomanagement, auf die Krisenentwicklung siehe Paul J.J. Welfens: Von der Griechenlandkrise zum Zerfall der Eurozone?, in: integration 3/2010, S. 264-273, hier S. 268-272. 
Versammlungen der Staats- und Regierungschefs der 17 Eurostaaten) zu entscheidenden Akteuren in der wirtschaftspolitischen Politikgestaltung geworden sind. ${ }^{5}$

\section{Europäische Reaktionen auf die erste Phase der Krise}

Im Sommer 2007 treten in Teilen des Finanzsystems vermehrt Ungleichgewichte zutage, die sich zu einer weltweiten Bankenkrise ausweiten. Zwischen August 2007 und September 2008 führt die Europäische Zentralbank (EZB) eine Reihe von temporären Maßnahmen ein, die dazu dienen, das Finanzsystem über die vermehrte Zufuhr von Liquidität funktionsfähig zu halten. Mit der Ausweitung der Krise - die US-Bank Lehmann Brothers hatte am 15. September 2008 Insolvenz angemeldet - kommen weitere Maßnahmen hinzu. Unter anderem verleiht die EZB zeitweise Zentralbankgeld ohne Mengenbeschränkung an Banken und reduziert die Bonitätsanforderungen für Wertpapiere, die dabei als Sicherheiten hinterlegt werden müssen. ${ }^{6}$

Die Regierungen antworten auf die Finanzkrise ihrerseits mit Bankenrettungs- und Konjunkturprogrammen. In vielen Fällen gelingt es ihnen damit, die Auswirkungen der Finanzkrise und des wirtschaftlichen Abschwungs im eigenen Land abzumildern. Der Preis dieser Programme sind jedoch ab 2009 erheblich steigende Defizite und Schuldenstände der öffentlichen Hand (siehe Tabelle 2). Darüber hinaus erwecken viele nationale Maßnahmen den Eindruck eines zunächst unkoordinierten Vorgehens. ${ }^{7}$

Tabelle 2: Staatliche Haushaltsdefizite und -überschüsse in 27 Mitgliedstaaten, 2006 bis 2011

\begin{tabular}{|l|r|r|r|r|r|r|}
\hline \multicolumn{1}{|r|}{ Staat } & $\mathbf{2 0 0 6}$ & $\mathbf{2 0 0 7}$ & $\mathbf{2 0 0 8}$ & $\mathbf{2 0 0 9}$ & $\mathbf{2 0 1 0}$ & $\mathbf{2 0 1 1} \mathbf{1}$ \\
\hline Belgien & 0,1 & $-0,3$ & $-1,3$ & $-5,8$ & $-4,1$ & $-3,6$ \\
\hline Bulgarien & 1,9 & 1,2 & 1,7 & $-4,3$ & $-3,1$ & $-2,5$ \\
\hline Dänemark & 5,2 & 4,8 & 3,2 & $-2,7$ & $-2,6$ & $-4,0$ \\
\hline Deutschland & $-1,6$ & 0,2 & $-0,1$ & $-3,2$ & $-4,3$ & $-1,3$ \\
\hline Estland & 2,5 & 2,4 & $-2,9$ & $-2,0$ & 0,2 & 0,8 \\
\hline Finnland & 4,0 & 5,3 & 4,3 & $-2,5$ & $-2,5$ & $-1,0$ \\
\hline Frankreich & $-2,3$ & $-2,7$ & $-3,3$ & $-7,5$ & $-7,1$ & $-5,8$ \\
\hline Griechenland & $-5,7$ & $-6,5$ & $-9,8$ & $-15,8$ & $-10,6$ & $-8,9$ \\
\hline Irland & 2,9 & 0,1 & $-7,3$ & $-14,2$ & $-31,3$ & $-10,3$ \\
\hline Italien & $-3,4$ & $-1,6$ & $-2,7$ & $-5,4$ & $-4,6$ & $-4,0$ \\
\hline Lettland & $-0,5$ & $-0,4$ & $-4,2$ & $-9,7$ & $-8,3$ & $-4,2$ \\
\hline
\end{tabular}

5 Als Übersicht über die beteiligten Akteure siehe Abbildung 1. Eine Übersicht über die Tagungen des Europäischen Rates bietet Gesa-Stefanie Brincker/Wolfgang Wessels: Europäischer Rat, in: Werner Weidenfeld/Wolfgang Wessels (Hrsg.): Jahrbuch der Europäischen Integration 2011, Baden-Baden 2012, S. 63-72.

6 Einen Überblick über die Maßnahmen der EZB bietet European Central Bank: The ECB's Response to the Financial Crisis, in: European Central Bank: Monthly Bulletin 10/2010, S. 51-74.

7 Vgl. James K. Jackson: The financial crisis: impact and response by the European Union. Congressional Research Service, 2009, S. 6. Andere Autoren stellen der koordinierten Vorgehensweise der Europäischen Union ein besseres Zeugnis aus, listen aber dennoch eine Reihe von höchst unterschiedlichen nationalen Maßnahmen auf. Vgl. Maria Grazia Attinasi: Euro Area Fiscal Policies: Response to the Financial Crisis, in: Ad van Riet (Hrsg.): Euro Area Fiscal Policies and the Crisis, European Central Bank: Occasional Paper No. 109, April 2010, S. 12-21, hier S. 13. 


\begin{tabular}{|c|c|c|c|c|c|c|}
\hline Staat & 2006 & 2007 & 2008 & 2009 & 2010 & $2011^{1}$ \\
\hline Litauen & $-0,4$ & $-1,0$ & $-3,3$ & $-9,5$ & $-7,0$ & $-5,0$ \\
\hline Luxemburg & 1,4 & 3,7 & 3,0 & $-0,9$ & $-1,1$ & $-0,6$ \\
\hline Malta & $-2,8$ & $-2,4$ & $-4,6$ & $-3,7$ & $-3,6$ & $-3,0$ \\
\hline Niederlande & 0,5 & 0,2 & 0,5 & $-5,6$ & $-5,1$ & $-4,3$ \\
\hline Österreich & $-1,5$ & $-0,9$ & $-0,9$ & $-4,1$ & $-4,4$ & $-3,4$ \\
\hline Polen & $-3,6$ & $-1,9$ & $-3,7$ & $-7,3$ & $-7,8$ & $-5,6$ \\
\hline Portugal & $-4,1$ & $-3,1$ & $-3,6$ & $-10,1$ & $-9,8$ & $-5,8$ \\
\hline Rumänien & $-2,2$ & $-2,9$ & $-5,7$ & $-9,0$ & $-6,9$ & $-4,9$ \\
\hline Schweden & 2,3 & 3,6 & 2,2 & $-0,7$ & 0,2 & 0,9 \\
\hline Slowakei & $-3,2$ & $-1,8$ & $-2,1$ & $-8,0$ & $-7,7$ & $-5,8$ \\
\hline Slowenien & $-1,4$ & 0 & $-1,9$ & $-6,1$ & $-5,8$ & $-5,7$ \\
\hline Spanien & 2,4 & 1,9 & $-4,5$ & $-11,2$ & $-9,3$ & $-6,6$ \\
\hline Tschechien & $-2,4$ & $-0,7$ & $-2,2$ & $-5,8$ & $-4,8$ & $-4,1$ \\
\hline Ungarn & $-9,3$ & $-5,1$ & $-3,7$ & $-4,6$ & $-4,2$ & 3,6 \\
\hline Vereinigtes Königreich & $-2,7$ & $-2,7$ & $-5,0$ & $-11,5$ & $-10,3$ & $-9,4$ \\
\hline Zypern & $-1,2$ & 3,5 & 0,9 & $-6,1$ & $-5,3$ & $-6,7$ \\
\hline $\begin{array}{l}\text { Europäische Union } \\
\text { (27 Staaten) }\end{array}$ & $-1,5$ & $-0,9$ & $-2,4$ & $-6,9$ & $-6,6$ & $-4,7$ \\
\hline \multicolumn{7}{|c|}{$\begin{array}{l}\text { Für das Jahr } 2011 \text { sind die Werte eine Schätzung. } \\
\text { Nettoneuverschuldung (-) beziehungsweise Nettohaushaltsüberschuss (+) in Prozent des Bruttoin- } \\
\text { landsproduktes zu Marktpreisen. }\end{array}$} \\
\hline
\end{tabular}

Quelle: AMECO-Datenbank der Europäischen Kommission, November 2011.

Am 12. Oktober 2008 treffen sich die Staats- und Regierungschefs der Eurozone in Paris. Dieser kurzfristig einberufene Euro-Gipfel ${ }^{8}$ stellt insofern ein Novum in der Integrationsgeschichte dar, als es bislang hochrangige Treffen der Eurostaaten nur informell auf Ebene der Finanzminister in der sogenannten Euro-Gruppe gegeben hatte. (siehe Abbildung 1) Der Gipfel erklärt, bestätigt durch den nachfolgenden Europäischen Rat, ${ }^{9}$ den Willen zu einem konzertierten Vorgehen gegen die Krise.

Die Europäische Kommission stößt ihrerseits Reformschritte in der EU-Finanzmarktaufsicht an. Hierzu richtet sie Ende Oktober 2008 eine Expertengruppe unter der Leitung von Jacques de Larosière ein, deren Bericht die Grundlage für eine institutionelle Reform der bestehenden Aufsichtsstrukturen bildet. ${ }^{10}$ Kurzfristig sollen jährliche EU-weite ,Stresstests“ für Banken dazu beitragen, das Vertrauen innerhalb des europäischen Finanzsystems wieder herzustellen.

8 Französische Ratspräsidentschaft: Gipfeltreffen der Länder der Eurozone: unter den Ländern der Eurozone abgestimmte Erklärung zum Aktionsplan, 12.10.2008, abrufbar unter: http://www.eu2008.fr/PFUE/lang/de/accueil/PFUE-10_2008/PFUE-12.10.2008/sommet_pays_zone_euro_declaration_plan_action_concertee.html (letzter Zugriff: 21.01.2010).

9 Rat der Europäischen Union: Tagung des Europäischen Rats vom 15. und 16. Oktober 2008 in Brüssel. Schlussfolgerungen des Vorsitzes, Dok. 14368/08.

10 High Level Group on Financial Supervision in the EU. Chaired by Jacques de Larosière. Report, Brüssel 2009. 


\section{Von der Finanzkrise zur Schuldenkrise}

Die Veröffentlichung der Ergebnisse des ersten Bankenstresstests am 1. Oktober 2009, dessen positives Ergebnis mit Erleichterung aufgenommen wird, ${ }^{11}$ fällt eng zusammen mit einem der Ausgangspunkte der zweiten Phase der Krise: Nach Bankbilanzen rücken nun Staatshaushalte in den Vordergrund. Am 20. Oktober 2009 veröffentlicht die neugewählte griechische Regierung drastisch nach oben korrigierte Defizitzahlen. ${ }^{12}$ Bereits bestehende Zweifel an der langfristigen Zahlungsfähigkeit Athens und weiterer Eurostaaten wachsen, was sich in schlechteren Bewertungen seitens der Ratingagenturen und steigenden Schuldzinsen niederschlägt. Wenn auch Spekulationsgeschäfte an den Finanzmärkten für diese Entwicklung nicht unbedingt ursächlich sind, so verstärken sie die negativen Effekte und erschweren eine Abschätzung der Risiken. Selbst ein kleines Land wie Griechenland kann so zu einer Gefahr für die Eurozone werden. Steigende Zinsen auf Staatsanleihen und steigende Preise für Versicherungen gegen Kreditausfall (sogenannte ,Credit Default Swaps') bilden in der Folge die Fieberkurven der Staatsschuldenkrise.

Ein erster informeller EU-Krisengipfel zu Griechenland findet im Februar 2010 statt. $^{13}$ Die 27 Staats- und Regierungschefs erklären, dass die Eurostaaten ,im Bedarfsfall entschlossen und koordiniert"14 handeln werden, um die Finanzmarktstabilität in der Eurozone zu sichern. Gleichzeitig unterstreichen sie, dass Griechenland nicht um finanzielle Unterstützung gebeten hat, sondern stärker sparen wird. Diese Formulierung entspricht noch dem Geist der Gründungsväter der WWU, nach dem die Gemeinschaft der Eurostaaten nicht für Schulden einzelner Staaten haftet. Doch bereits in den folgenden Wochen verschärft sich die Situation Griechenlands dramatisch. Im Rahmen des Europäischen Rates am 25. und 26. März 2010 in Brüssel konkretisieren die Staats- und Regierungschefs der Eurozone in einer gemeinsamen Erklärung die Grundprinzipien einer finanziellen Rettungsaktion: Um die Stabilität der Eurozone als Ganzes zu gewährleisten, verpflichten sich ihre Mitglieder zu koordinierten bilateralen Darlehen, falls sich Griechenland an den Finanzmärkten nicht mehr refinanzieren kann. ${ }^{15}$ Der Beschluss hierzu soll einstimmig erfolgen. Auch Mittel des Internationalen Währungsfonds (IWF), dessen Einbindung unter anderem die EZB lange skeptisch gegenüber stand, ${ }^{16}$ sind Teil des gemeinsamen Maßnahmenkatalogs.

Der Europäische Rat beauftragt seinerseits seinen Präsidenten Herman Van Rompuy mit der Bildung einer Arbeitsgruppe ,Wirtschaftspolitische Steuerung '. ${ }^{17}$ Die , Task Force ' soll bis zum Ende des Jahres 2010 Vorschläge vorlegen, um das ,Ziel eines verbesserten Krisenbewältigungsrahmens und einer besseren Haushaltsdisziplin zu erreichen“.18

\section{Ein Hilfspaket für Griechenland, ein Rettungsschirm für den Euro}

Am 12. April 2010 präsentieren die Euro-Finanzminister Details eines Hilfspakets für Griechenland. Sie stellen bis zu 30 Milliarden Euro an Notkrediten für Griechenland in Aus-

11 Vgl. Nikki Tait: EU big banks pass capital stress tests, in: Financial Times, 02.10.2009.

12 Werner Mussler/Rainer Herrmann: Griechenlands Defizit plötzlich verdoppelt, in: Frankfurter Allgemeine Zeitung, 21.10.2009.

13 Informelles Treffen der Staats- und Regierungschefs der Europäischen Union, Brüssel, den 11. Februar 2010.

14 Ebenda.

15 Erklärung der Staats- und Regierungschefs der Mitgliedstaaten des Euro-Währungsgebiets, Brüssel, den 25. März 2010, S. 1.

16 Mark Schieritz: Der Vertrag wird nicht verletzt, Interview mit Lorenzo Bini Smaghi, in: Die Zeit, 25.03.2010.

17 Europäischer Rat: Tagung des Europäischen Rats vom 25. und 26. März 2010 in Brüssel. Schlussfolgerungen des Vorsitzes, EUCO 7/10, S. 6.

18 Ebenda. 
sicht, der IWF bis zu 15 Milliarden Euro. Elf Tage später ruft Griechenland die Hilfen ab. Das am 2. Mai 2010 zugesagte Paket umfasst bis zu 80 Milliarden Euro seitens der Eurozone und bis zu 30 Milliarden Euro seitens des IWF. Im Gegenzug verpflichtet sich Griechenland zu einem harten Reform- und Sparprogramm, das auf europäischer Seite durch Kommission und EZB überwacht wird. Zusammen mit dem IWF bilden die beiden Institutionen die sogenannte Troika.

Bei den Hilfen für in Schieflage geratene Eurostaaten handelt es sich um Kredite, die als solche prinzipiell zurückgezahlt werden müssen. Vereinzelt wird daher sogar auf Gewinne für die Geberstaaten aus den zu zahlenden Schuldzinsen spekuliert. ${ }^{19}$ Letztendlich tragen jedoch die Staatshaushalte und damit die Steuerzahler in der Eurozone das Risiko eines Zahlungsausfalls. Vor dem Hintergrund der Budgethoheit nationaler Parlamente (wie sie auch das Bundesverfassungsgericht in einem späteren Urteil zu den Rettungsmaßnahmen nochmals unterstreicht), ${ }^{20}$ muss daher deren Zustimmung für das Griechenland-Rettungspaket eingeholt werden. In Deutschland billigen Bundestag und Bundesrat die Hilfen am 7. Mai $2010^{21}$ und machen damit den Weg für einen formellen Beschluss der Staats- und Regierungschefs der Eurozone an diesem Tag frei. ${ }^{22}$ Angesichts von immer stärkeren Marktverwerfungen und auf eindringliches Anraten des damaligen EZB-Präsidenten Jean-Claude Trichet, der eine Systemkrise befürchtet, verabschieden die Staats- und Regierungschefs der Eurozone in einer Nachtsitzung vom 7. auf den 8. Mai 2010 jedoch nicht nur ein Rettungspaket für Griechenland, sondern einen europäischen Stabilisierungsmechanismus, einen ,Rettungsschirm‘ für die gesamte Eurozone. ${ }^{23}$

In den Morgenstunden des 10. Mai 2010 einigen sich die EU-Finanzminister in einer auBerordentlichen Ratssitzung auf die Details des Rettungsschirms. ${ }^{24} \mathrm{Zu}$ Kreditgarantien der Eurostaaten in Höhe von 440 Milliarden Euro kommen Kreditzusagen in Höhe von 60 Milliarden Euro aus gemeinschaftlichen Mitteln der Europäischen Union. Der IWF sagt später weitere 250 Milliarden Euro zu.

Die zwischenstaatlich angelegten Kreditgarantien der Eurostaaten werden über ein spezielles Investmentvehikel mit Sitz in Luxemburg bereitgestellt, die auf drei Jahre angelegte „European Financial Stability Facility“ (EFSF). Diese kann am Kapitalmarkt zu günstigen Konditionen Kredite aufnehmen und an Krisenstaaten weiterreichen. Die Eurostaaten garantieren die von der EFSF vergebenen Kredite zu einem festen Anteil. Um sicherzustellen, dass die EFSF von Rating-Agenturen die Bestnote erhält und damit von niedrigen Zinsen profitiert, ist die tatsächliche Kapazität allerdings niedriger als die Garantiesumme von 440 Milliarden Euro. Das gemeinschaftliche Element des Rettungsschirmes bilden Kredite aus dem EU-Haushalt im Rahmen eines Programms, das als „European Financial Stabilisation

19 Christoph Hein: Regling: Irlands Rettung ist für Deutschland wahrscheinlich ein Gewinn, in: Frankfurter Allgemeine Zeitung, 02.12.2010; Schieritz: Der Vertrag wird nicht verletzt, 2010.

20 Bundesverfassungsgericht: Leitsätze zum Urteil des Zweiten Senats vom 07.09.2011, 2 BvR 987/10, 2 BvR 1485/10, 2 BvR 1099/10.

21 Gesetz zur Übernahme von Gewährleistungen zum Erhalt der für die Finanzstabilität in der Währungsunion erforderlichen Zahlungsfähigkeit der Hellenischen Republik (Währungsunion-Finanzstabilitätsgesetz - WFStG) vom 7. Mai 2010, Bundesgesetzblatt Teil I, Nr. 19, S. 537.

22 Zur Beteiligung des Deutschen Bundestages an den ersten Hilfsmaßnahmen für Griechenland im Lichte der gestärkten Rechte nach dem Urteil des Bundesverfassungsgerichts zum Vertrag von Lissabon siehe: Sebastian Gröning-von Thüna: Die neuen Begleitgesetze zum Vertrag von Lissabon aus Sicht des Deutschen Bundestages - offene Fragen und neue Herausforderungen, in: integration 4/2010, S. 312-333, hier S. 331-332.

23 Erklärung der Staats- und Regierungschefs der Mitgliedstaaten des Euro-Währungsgebiets, Brüssel, 7. Mai 2010, abgedruckt in: Generalsekretariat des Rates (Hrsg.): Der Europäische Rat 2010, Luxemburg 2011, S. 29.

24 Rat der Europäischen Union: Mitteilungen an die Presse. Außerordentliche Tagung des Rats. Wirtschaft und Finanzen, Dok. 9596/10. 
Mechanism“ (EFSM) bezeichnet wird. Das Programm basiert auf Art. 122 Abs. 2 des Vertrags über die Arbeitsweise der Europäischen Union (AEUV) und erlaubt, von ,,außergewöhnlichen Ereignissen, die sich seiner Kontrolle entziehen“ betroffene Mitgliedstaaten finanziell zu unterstützen.

Bundestag und Bundesrat billigen den Beitrag Deutschlands zum Rettungsschirm am 21. Mai 2010.25

Ebenfalls am 10. Mai 2010 beginnt die EZB, Staatsanleihen labiler Eurostaaten zu kaufen, um deren Refinanzierungskosten zu senken. Sie begründet das umstrittene Kaufprogramm damit, dass sie ihre eigentliche Aufgabe - die Durchführung der Geldpolitik - in den betroffenen Staaten andernfalls nicht erfüllen könne. Dass solche der geldpolitischen Orthodoxie widersprechende Maßnahmen intern stark umstritten sind, zeigt sich dem Vernehmen nach auch in den späteren Rücktritten zweier deutscher Zentralbanker: Bundesbankpräsident Axel Weber (11. Februar 2011) und EZB-Direktoriumsmitglied Jürgen Stark (9. September 2011). ${ }^{26}$

\section{Reform des wirtschaftspolitischen Regierens: Entwürfe und Beschlüsse}

Neben der Bereitstellung von Krediten, die in erster Linie Krisensymptome bekämpfen, verfolgen die Akteure auf EU-Ebene verschiedene Maßnahmen zur Stabilisierung und Reform der Haushalts- und Wirtschaftspolitiken in der Eurozone. Im September 2010 stellt die Europäische Kommission ein Paket von sechs Gesetzgebungsvorschlägen im Bereich des wirtschaftspolitischen Regierens vor (bezeichnet als Sixpack). ${ }^{27}$ Das Paket, das auch Vorschläge der Task Force zur wirtschaftspolitischen Steuerung unter Van Rompuy aufgreift, umfasst zum einen eine Reform der fiskalpolitischen Regeln des Stabilitäts- und Wachstumspaktes. Zum anderen soll es die stärkere Koordinierung nationaler Wirtschaftspolitiken ermöglichen.

Im gleichen Monat beschließt der Ministerrat einen als „Europäisches Semester“ bezeichneten reformierten Zyklus zur Koordinierung der Wirtschaftspolitik mit dem Europäischen Rat als Schlüsselinstanz. ${ }^{28}$

$\mathrm{Zu}$ einem weiteren gravierenden Projekt fordern Angela Merkel und Nicolas Sarkozy nach einem Treffen in Deauville am 18. Oktober 2010 auf: Eine Vertragsänderung soll einen permanenten Mechanismus zur Rettung insolventer Staaten erlauben. ${ }^{29}$ Obwohl das deutsch-französische Vorgehen als Hinterzimmerpolitik kritisiert wird, ${ }^{30}$ einigt sich der Europäische Rat am 28. und 29. Oktober 2010 auf eine begrenzte Änderung der EU-Verträge zur Schaffung eines ,ständigen Krisenmechanismus zur Wahrung der Finanzstabilität des Euro-Währungsgebiets insgesamt““.31 Am 16. und 17. Dezember 2010 leitet er eine entsprechende Ergänzung von Art. 136 AEUV ein, der einen zusätzlichen Absatz erhält: „Die Mit-

25 Gesetz zur Übernahme von Gewährleistungen im Rahmen eines europäischen Stabilisierungsmechanismus vom 22.05.2010, Bundesgesetzblatt Teil I, Nr. 24, S. 627.

26 Stefan Ruhkamp: Die Hüter des Grundsatzstreits, in: Frankfurter Allgemeine Zeitung, 01.11.2011.

27 Europäische Kommission: Wirtschaftspolitische Steuerung in der EU: Kommission legt umfassendes Legislativpaket vor, Pressemitteilung IP/10/1199.

28 Rat der Europäischen Union: Mitteilungen an die Presse. 3030. Tagung des Rates. Wirtschaft und Finanzen, Dok. 13161/10.

29 Französische Regierung: Franco-German Declaration. Statement for the France-Germany-Russia Summit, Deauville, 18.10.2010, abrufbar unter: http://www.elysee.fr/president/root/bank_objects/Franco-german_decla ration.pdf (letzter Zugriff: 06.06.2011).

30 Guy Dinmore: Frattini shuns Paris-Berlin pre-cooked deals, in: Financial Times, 05.11.2010.

31 Europäischer Rat: Tagung des Europäischen Rats vom 28. und 29. Oktober 2010 in Brüssel. Schlussfolgerungen, EUCO 25/1/10, S. 2. 
gliedstaaten, deren Währung der Euro ist, können einen Stabilitätsmechanismus einrichten, der aktiviert wird, wenn dies unabdingbar ist, um die Stabilität des Euro-Währungsgebiets insgesamt zu wahren. Die Gewährung aller erforderlichen Finanzhilfen im Rahmen des Mechanismus wird strengen Auflagen unterliegen“.32 Der „Europäische Stabilitätsmechanismus“ (ESM) soll ab Juli 2013 die beiden temporären Stabilisierungsinstrumente, EFSF und EFSM, ablösen. Die faktische Abkehr von einem Grundprinzip der WWU, nach dem Mitgliedstaaten nicht gegenseitig für ihre Schulden haften (Art. 125 AEUV), erhält damit ihren formalen Rahmen. ${ }^{33}$

Bereits zwei Wochen zuvor, am 28. November 2010, erhielt Irland aufgrund seines aus dem Ruder laufenden Staatsdefizits als erstes Land einen Notkredit des temporären EuroRettungsschirms. Der EFSM stellt 22,5 Milliarden Euro, der EFSF und - bilateral - Großbritannien, Schweden und Dänemark stellen 22,5 Milliarden Euro sowie der IWF stellt 22,5 Milliarden Euro zur Verfügung. ${ }^{34}$

\section{Euro-Plus-Pakt und Europäischer Stabilitätsmechanismus}

Die bisher erfolgten Schritte sind nach wie vor nicht ausreichend, um die Finanzmärkte zu beruhigen. Wieder sehen sich die 17 Staats- und Regierungschefs der Eurozone zu Notmaßnahmen der Brandbekämpfung gezwungen und kommen am 11. März 2011 im Rahmen einer außerordentlichen Sitzung des Europäischen Rates zu einem Sondergipfel zusammen. ${ }^{35}$ Sie beschließen eine Erhöhung der Garantiemittel für die EFSF auf insgesamt 780 Milliarden Euro, sodass dieser Kredite in Höhe von 440 Milliarden Euro vergeben kann. Außerdem verabschieden sie einen „Pakt für den Euro“, später als „Euro-Plus-Pakt“ bezeichnet. ${ }^{36}$ Der auf eine deutsche Initiative zurückgehende Pakt sieht vor, dass sich die Staatsund Regierungschefs jährlich zu konkreten Maßnahmen zur Steigerung von Wettbewerbsfähigkeit und Konvergenz verpflichten. ${ }^{37}$ Deren Umsetzung soll von der Kommission überprüft werden. Sanktionen sind allerdings nicht vorgesehen. Die engere Koordinierung der Wirtschaftspolitik im Rahmen des Paktes steht auch denjenigen EU-Mitgliedstaaten offen, die den Euro nicht eingeführt haben. Bulgarien, Dänemark, Lettland, Litauen, Polen und Rumänien entscheiden sich für eine Teilnahme. Der unvollständige Teilnehmerkreis deutet an, dass der Gemeinschaftscharakter des Euro-Plus-Paktes und seine Stellung im Gefüge des wirtschaftspolitischen Regierens nicht eindeutig sind. ${ }^{38}$

Der Europäische Rat selbst tagt am 24. und 25. März 2011 und verabschiedet auf Basis der Beschlüsse vom 11. März ein ,umfassendes“ Maßnahmenpaket zur Bekämpfung der Krise. ${ }^{39}$ Insbesondere nehmen die Staats- und Regierungschefs die Vertragsänderung zur Einrichtung des ESM an. Der ESM soll auf 80 Milliarden Euro Grundkapital zurückgreifen

32 Europäischer Rat: Tagung des Europäischen Rats vom 16. und 17. Dezember 2010 in Brüssel. Schlussfolgerungen, EUCO 30/1/10, Anlage I.

33 Vgl. dazu Ulrich Hufeld: Zwischen Notrettung und Rütlischwur: der Umbau der Wirtschafts- und Währungsunion in der Krise, in: integration 2/2011, S. 117-131.

34 Rat der Europäischen Union: Statement by the Eurogroup and ECOFIN Ministers, Dok. 118051/10.

35 Schlussfolgerungen der Staats- und Regierungschefs der Mitgliedstaaten des Euro-Währungsgebiets vom 11. März 2011, Brüssel, den 11. März 2011.

36 Vgl. zum Euro-Plus-Pakt Daniel Gros/Cinzia Alcidi: Was bringt der „Euro-plus-Pakt“"?, in: integration 2/2011, S. 164-171.

37 Vgl. Europäischer Rat: Tagung des Europäischen Rats vom 24. und 25. März 2011 in Brüssel. Schlussfolgerungen, EUCO 10/1/11, Annex I.

38 The Economist: The divisiveness pact, 10.03.2011.

39 Europäischer Rat: Tagung des Europäischen Rats vom 24. und 25. März 2011. Schlussfolgerungen, EUCO 10/ 1/11 REV 1 
können, das die Eurostaaten ab 2013 einzahlen. Hinzu kommen Kreditgarantien in Höhe von 620 Milliarden Euro. Ähnlich wie bei der EFSF müssen Kreditmittel des ESM zur Erlangung eines bestmöglichen Ratings , übersichert ' werden, sodass seine effektive Kapazität mit 500 Milliarden Euro niedriger liegt. Ergänzt wird die Kapazität des ESM um bis zu 250 Milliarden Euro, die weiterhin der IWF bereitstellt.

\section{Ein neuer Flächenbrand droht}

Am 17. Mai 2011 erhält mit Portugal der zweite Eurostaat Hilfen des Rettungsschirms. Das Hilfspaket umfasst bis zu 78 Milliarden Euro. ${ }^{40}$ EFSF, EFSM und der IWF sagen jeweils bis zu 26 Milliarden Euro zu. Portugal nimmt im Gegenzug wesentliche Schritte zum Schuldenabbau vor.

Obwohl zu diesem Zeitpunkt erst ein Teil der Kapazität des Rettungsschirms vergeben ist, wird immer öfter die Frage gestellt, ob der Umfang ausreicht, um im Fall einer möglichen Ansteckung weiterer Staaten reagieren zu können. Im Europäischen Rat einigen sich die Geberstaaten Mitte Juni 2011 auf eine Erhöhung der Kreditgarantien auf 780 Milliarden Euro, sodass die EFSF künftig seine angestrebte Kapazität von 440 Milliarden Euro ausschöpfen kann. ${ }^{41}$ Die 17 Staats- und Regierungschefs der Eurozone erweitern darüber hinaus am 21. Juli 2011 die Kompetenzen der EFSF. Unter anderem soll die EFSF die Möglichkeit erhalten, unter strengen Auflagen am sekundären Anleihenmarkt zu intervenieren. ${ }^{42}$

Gleichzeitig verabschieden sie ein zweites Hilfspaket für Griechenland. Die Bankwirtschaft erklärt sich zu einem Verzicht auf 21 Prozent der ausstehenden Forderungen an den griechischen Staat bereit. Diese Beteiligung privater Gläubiger ist eine der zentralen Konfliktlinien der Krise: Einerseits erscheint es gerecht, die mit Steuergeldern geretteten Banken an den Verlusten eines unausweichlichen Schuldenschnitts für Griechenland zu beteiligen. Andererseits stellt ein Rückzahlungsausfall einen Vertragsbruch dar, der die Kreditwürdigkeit von Staaten generell infrage stellt. Hohe Risikoaufschläge auf die Verzinsung von Staatsanleihen wären die Folge. Hinzu kommt die Angst vor einer Kettenreaktion an den Finanzmärkten, wenn in diesem Szenario Kreditausfallversicherungen greifen, mit denen unreguliert Spekulationsgeschäfte betrieben wurden. Daher erhält die Beteiligung der Banken das Etikett ,freiwillig'.

Die EZB nimmt Marktbeobachtern zufolge am 4. August 2011 mit dem Kauf von irischen und portugiesischen Staatsanleihen ihren Versuch wieder auf, die Marktzinsen dieser Papiere zu senken. ${ }^{43}$ Drei Tage später kündigt sie kaum verklausuliert an, ihr Ankaufprogramm auf spanische und italienische Papiere auszudehnen. ${ }^{44}$ Die Solvenz Italiens, der drittgrößten Volkswirtschaft der Eurozone, geriet in den Monaten zuvor mehr und mehr in den Blickpunkt, sodass sich die EZB am 5. August 2011 zu einem vertraulichen Brief an die italienische Regierung veranlasst sieht, der mehr Anstrengungen bei der Haushaltskonsolidierung fordert. ${ }^{45}$ Aber auch Griechenland verschwindet nicht aus den Schlagzeilen. Die Troika aus Kommission, EZB und IWF setzt am 2. September 2011 kurzzeitig ihre Überwachungs-

40 Rat der Europäischen Union: Mitteilungen an die Presse. 3088. Tagung des Rates. Wirtschaft und Finanzen, Dok. 10191/11.

41 Europäischer Rat: Tagung des Europäischen Rats vom 23. und 24. Juni 2011 in Brüssel. Schlussfolgerungen, EUCO 23/1/11.

42 Rat der Europäischen Union: Erklärung der Staats- und Regierungschefs des Euro-Währungsgebietes und der EU-Organe, Brüssel, 21. Juli 2011.

43 Financial Times: Investors criticise ECB step on bonds, 06.08.2011.

44 Europäische Zentralbank: Statement by the President of the ECB, Pressemitteilung, 07.08.2011.

45 Tobias Piller: Drakonische Forderung von Trichet und Draghi an Italien, in: Frankfurter Allgemeine Zeitung, 01.10.2011. 
mission aus. Als Begründung werden Verzögerungen bei der Umsetzung des griechischen Reformprogramms angeführt.

\section{Reform des wirtschaftspolitischen Regierens: Implementierung des Sixpacks}

Ein Jahr nach der Vorstellung des Entwurfs seitens der Kommission nehmen Europäisches Parlament (28. September 2011) und Ministerrat (4. Oktober 2011) das sogenannte Sixpack zur Reform der fiskalpolitischen Regeln des Stabilitäts- und Wachstumspaktes sowie des wirtschaftspolitischen Regierens in der Europäischen Union an. ${ }^{46}$

In der fiskalpolitischen Überwachung soll die maximal erlaubte Erhöhung von Ausgaben an das Wirtschaftswachstum gekoppelt werden. Gibt ein Staat mehr aus, als diese Relation erlaubt, sind Sanktionen möglich. Die Korrektur fiskalischer Fehlentwicklungen soll sich auf den Abbau des Schuldenstands konzentrieren und damit langfristiger orientiert sein. Feiner abgestufte finanzielle Sanktionen setzen früher ein, wenn der Ministerrat auf Empfehlung der Kommission ein haushaltspolitisches Fehlverhalten feststellt. Anders als zuvor ist dabei eine ausdrückliche Entscheidung des Rates gegen Sanktionen notwendig, um sie zu verhindern.

Die stärkere Überwachung und gegebenenfalls Korrektur nationaler Wirtschaftspolitiken soll zur Vermeidung makroökonomischer Ungleichgewichte beitragen. Ausgehend von einer ähnlichen Konstruktion in der Fiskalpolitik wird ein „Verfahren bei einem übermäßigen Ungleichgewicht“ eingeführt, das gegenüber Eurostaaten, die die Vorgaben nicht einhalten, sanktionsbehaftet durchgesetzt werden kann. EU-Institutionen, so auch die Europäische Kommission, erhalten damit mehr Einfluss in der Wirtschaftspolitik, die bisher weitgehend in die Zuständigkeit der Mitgliedstaaten fiel.

\section{Jüngste Entwicklungen: kein Ende der Krise in Sicht}

Als im Spätsommer 2011 große Volkswirtschaften der Eurozone wie Italien und Frankreich ins Visier der Märkte zu geraten scheinen, spitzt sich die Lage erneut dramatisch zu. Am 23. Oktober 2011 kommen die Staats- und Regierungschefs der Europäischen Union und der Eurozone zu einem Doppelgipfel zusammen. ${ }^{47}$ Die eigentliche Beschlussfassung wird auf einen unmittelbar folgenden Gipfel am 26. Oktober 2011 vertagt. Dort beschließen die 17 Staats- und Regierungschefs der Eurozone wiederum in einer Nachtsitzung ein weiteres ,umfassendes * Maßnahmenpaket zur Stabilisierung der Eurozone, das folgende Elemente enthält::48 (1) private Gläubiger Griechenlands verzichten freiwillig auf 50 Prozent ihrer Forderungen, (2) die verbleibende Kapazität der EFSF in Höhe von rund 250 Milliarden Euro wird durch sogenannte Hebel auf das Vier- bis Fünffache verstärkt, (3) Rekapitalisierung des europäischen Bankensystems. Auch wenn das Verhandlungsergebnis substanziell ist, bleiben Beobachter angesichts der bisherigen Erfahrungen skeptisch. Ebenso wenig wie die finanziellen Zusagen vermag die Ankündigung von ,,[z]ehn Maßnahmen zur Verbesserung der wirtschaftspolitischen Steuerung im Euro-Währungsgebiet“ die Märkte zu beruhigen, die der Eurozone mehr Möglichkeiten geben, sie betreffende Entscheidungen unabhängig von der institutionellen Struktur der Europäischen Union abzustimmen. Dazu werden die Ad-hoc-Gipfeltreffen der Staats- und Regierungschefs der Eurozone verstetigt. Sie finden zukünftig mindestens zweimal jährlich statt und erhalten einen selbstständigeren administrativen Unterbau (siehe Abbildung 1).

46 Rat der Europäischen Union: Mitteilungen an die Presse. 3115. Tagung des Rates. Wirtschaft und Finanzen, Dok. 14890/11. Im Einzelnen umfasst das Paket fünf Verordnungen und eine Richtlinie, abgedruckt im Amtsblatt der EU, Nr. L 306 vom 23.11.2011, S. 1-47.

47 Europäischer Rat: Europäischer Rat 23. Oktober 2011. Schlussfolgerungen, EUCO 52/1/11.

48 Erklärung des Euro-Gipfels, 26.10.2011. 
Abbildung 1: Institutionelle Architektur der ,economic governance

\begin{tabular}{|l|}
\hline EUROPÄISCHE UNION \\
\hline Europäischer Rat (EU-Gipfel) \\
27 Staats- und Regierungschefs, Präsident des Europäischen \\
Rates, Prä sident der Kommisson \\
\hline $\begin{array}{l}\text { Status quo: } \\
\text { - Leitliniengeber } \\
\text { - Treffen mind. 4x jährlich } \\
\text { - seit Ende 2009 hauptamtlicher } \\
\text { Präsident }\end{array}$ \\
\hline
\end{tabular}

\section{Ecofin-Rat}

27 Wirtschafts- und Finanzminister

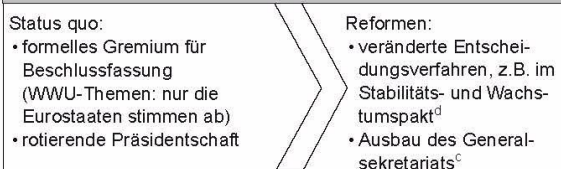

\section{Wirtschafts- und Finanzausschuss ${ }^{f}$}

max. 58 hochrangige Beamte aus den nationalen Ministerien und Zentralbanken, der Kommission, der EZB

$\left.\left.\begin{array}{|l}\hline \text { Status quo: } \\ \text { - eingerichtet 1999, bereitet } \\ \text { Ecofin vor } \\ \text { - wählt Mitglied als Präsidenten } \\ \text { - bei Kommission angesiedeltes, } \\ \text { aber eigenständiges Sekretariat }\end{array}\right\rangle>\begin{array}{l}\text { Reformen }^{c} \text { : } \\ \text { Ausbau Sekretariat }\end{array}\right\rangle$

\begin{tabular}{|l|l|} 
Euro-Gipfel \\
17 Staats- und Regierungschefs, Präsident des Euro-Gipfels ${ }^{\mathrm{b}}$, \\
Präsident der Kommisson
\end{tabular}

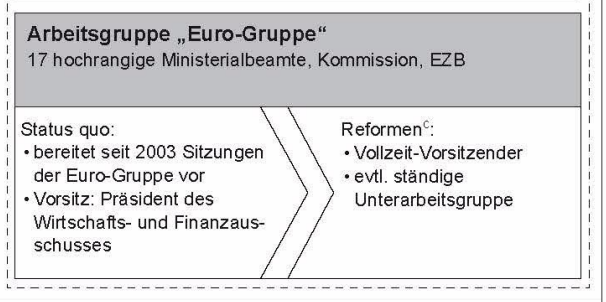

Die Präsidenten des Euro-Gipfels, der Kommission und der Euro-Gruppe treffen sich mind. 1x monatlich, der Präsident der EZB kann eingeladen werden. ${ }^{c}$

b Bis zur nächsten Wahl des Präsidenten des Europäischen Rates hat der gegenwärtige Präsident des Europäischen Rates den Vorsitz des Euro-Gipfels inne.

c Anlage 1, Erklärung des Euro-Gipfels vom 26. Oktober 2011.

d Rat der Europäischen Union: Mitteilung an die Presse. 3115. Tagung des Rates. Wirtschaft und Finanzen, Luxemburg, den 4. Oktober 2011, Dok. $14890 / 11$.

e Protokoll (Nr. 14) betreffend die Euro-Gruppe, in: Amtsblatt der EU, Nr. C 83 vom 30. März 2010, S. 283.

f Art. 134 AEUV; überarbeitete Satzung des Wirtschafts- und Finanzausschusses, in: Amtsblatt der EU, Nr. L 158 vom 27. Juni 2003, S. 58.

Tatsächlich wird der erreichte Kompromiss von unerwarteter Seite wieder infrage gestellt: Am 31. Oktober 2011 kündigt der damalige griechische Premierminister Giorgos Papandreou überraschend einen Volksentscheid über das Rettungspaket an. Nach heftigen Marktreaktionen und scharfer Kritik zieht er den Plan drei Tage später zurück. Regierungspartei und Opposition sollen stattdessen eine Regierung der nationalen Einheit bilden. Die Koalitionspartner einigen sich am 10. November 2011 auf den ehemaligen Vizepräsident der EZB, Lucas Papademos, als neuen Premierminister.

Der deutlichste Impuls zur Beruhigung der Finanzmärkte geht in dieser Periode aber von den Zentralbanken aus, die eines ihrer wirkungsvollsten Instrumente zum Einsatz bringen: Anfang November 2011 erleichtern die Zentralbanken Englands, Kanadas, Japans, der Schweiz, der USA und die EZB in einer abgestimmten Aktion den Zugang zu Liquidität, da sich die Geschäftsbanken untereinander immer weniger Geld leihen und - ähnlich wie auf dem Höhepunkt der Finanzkrise im Herbst 2008 - der Markt für Kredite auszutrocknen droht. ${ }^{49}$

49 Europäische Zentralbank: Coordinated central bank action to address pressures in global money markets, Pressemitteilung, 30.11.2011. 
Italien versucht derweil nach dem Rücktritt von Premierminister Silvio Berlusconi mit einer neuen Regierungsmannschaft aus Wirtschaftsfachleuten die notwendigen Reformen zu beschleunigen. Bereits Anfang Dezember 2011 legt der neue Premierminister Mario Monti ein ambitioniertes Sparpaket vor, das an den Börsen positiv aufgenommen wird. ${ }^{50}$

Vor dem Hintergrund dieser - zumindest kurzfristigen - Entspannung treten vom 8. bis 9. Dezember 2011 zunächst die Staats- und Regierungschefs der Eurozone und anschließend der Europäische Rat zusammen. Im Vorfeld sorgt die Ankündigung Merkels und Sarkozys für Aufmerksamkeit, auf der Basis eines deutsch-französischen Plans bereits im März 2012 eine Änderung der europäischen Verträge durchsetzen zu wollen - nötigenfalls auch nur mit den 17 Eurostaaten. ${ }^{51}$

Tatsächlich bildet die Trennlinie zwischen Eurostaaten und Nicht-Eurostaaten ein Schlüsselmoment dieses Gipfels - allerdings in einer Konstellation, mit der vorher kaum zu rechnen war und die zunächst sogar die inhaltlichen Ergebnisse des Euro-Gipfels in den Hintergrund drängt. Von zehn Nicht-Eurostaaten tragen neun den Plan einer Änderung der EU-Verträge zur Reform des Regelwerks der gemeinsamen Währung mit. ${ }^{52}$ Einzig der britische Premier David Cameron lehnt aufgrund fehlender Zugeständnisse an Großbritannien eine Vertragsänderung ab. Damit ist Großbritannien innerhalb der Europäischen Union isoliert. Die übrigen 26 EU-Mitgliedstaaten streben einen zwischenstaatlichen Vertrag außerhalb des Rahmens der Europäischen Union an, der einen Teil der beschlossenen Reformen mit hoher Verbindlichkeit verankern soll.

Inhaltlich verabschiedet der Euro-Gipfel unter dem Leitbild einer ,fiskalpolitischen Stabilitätsunion“"53 Maßnahmen in zwei Bereichen: Neue Elemente zur Stärkung der Architektur der WWU umfassen insbesondere verschärfte Haushaltsregeln, deren verbindliche Übernahme in nationales Verfassungsrecht vom Europäischen Gerichtshof überprüft werden kann. Der „,neue fiskalpolitische Pakt“ ergänzt die bereits im Oktober 2011 beschlossene verstärkte Koordinierung der Wirtschaftspolitik: „Die Vorschriften für das Verfahren bei einem übermäßigen Defizit (Artikel 126 AEUV) werden für die Mitgliedstaaten des EuroWährungsgebiets verschärft. Sobald die Kommission festgestellt hat, dass ein Mitgliedstaat die 3-\%-Schwelle überschritten hat, erfolgen automatisch Konsequenzen, es sei denn, die Mitgliedstaaten des Euro-Währungsgebiets sprechen sich mit qualifizierter Mehrheit dagegen aus. "54 Im Bereich der kurzfristigen Stabilisierungsinstrumente beschließen die Staatsund Regierungschefs, das Inkrafttreten des ESM nach Möglichkeit auf Juli 2012 vorzuziehen. Bis Juli 2013 soll er parallel mit der dann auslaufenden EFSF bestehen. Außerdem soll im März 2012 überprüft werden, ob die Gesamtausstattung von EFSF beziehungsweise ESM ausreichend ist. Als kurzfristige Maßnahme wird eine Erhöhung der bilateralen Kreditlinien von Mitgliedstaaten an den IWF um insgesamt bis zu 200 Milliarden Euro angeregt, die dieser wiederum zur Bekämpfung der Krise verwenden könnte. Die Regel, dass grundlegende Entscheidungen zum Einsatz des ESM einen einstimmigen Beschluss der Eurogruppe voraussetzen, wird durch die Möglichkeit zu Dringlichkeitsbeschlüssen mit reduziertem Quorum ergänzt. Vorausgesetzt, dass Kommission und EZB die finanzielle und wirtschaftliche Tragfähigkeit der Eurozone gefährdet sehen, genügt dann eine qualifizierte Mehrheit

50 Tobias Piller: Monti ohne Tabu, in: Frankfurter Allgemeine Zeitung, 06.12.2011.

51 Michaela Wiegel/Hendrik Kafsack: Merkel und Sarkozy wollen Vertragsänderung im März 2012, in: Frankfurter Allgemeine Zeitung, 06.12.2011.

52 Europäischer Rat: Erklärung der Staats- und Regierungschefs des Euro-Währungsgebiets, Brüssel, den 9. Dezember 2011, S. 7.

53 Ebenda, S. 2.

54 Ebenda, S. 3-4. 
von 85 Prozent (das Stimmgewicht jeden Staates wird anhand seines Anteils am Kapital des ESM gewichtet). Deutschland, Frankreich und Italien verfügen damit auch bei Dringlichkeitsbeschlüssen über ein Vetorecht. Der teilweise Forderungsverzicht privater Gläubiger als Voraussetzung für Notkredite, wie er im Fall Griechenlands erfolgte, wird nicht wie ursprünglich geplant festgeschrieben und damit unwahrscheinlich. ${ }^{55}$

Die Reaktion von Beobachtern und Finanzmärkten auf die Gipfelbeschlüsse fällt zurückhaltend aus. Zweifel bestehen insbesondere hinsichtlich der juristischen Durchsetzbarkeit einiger Neuerungen, zumal sich auch das rechtliche Verhältnis zwischen EU-Verträgen und der angestrebten zwischenstaatlichen Vereinbarung der 26 Staaten als problematisch erweisen könnte. ${ }^{56}$

Tabelle 3: Übersicht über die Bereitstellung und Zusage von Geldern und Garantien

\begin{tabular}{|c|c|c|c|c|c|}
\hline Datum $^{1}$ & Adressat & Mitgliedstaaten $^{2}$ & $\begin{array}{c}\text { Europäische } \\
\text { Union }\end{array}$ & IWF & EFSF \\
\hline 12.04 .2010 & $\begin{array}{l}\text { Zusagen für } \\
\text { Griechenland }\end{array}$ & $30 \mathrm{Mrd}$. Euro & & 15 Mrd. Euro & \\
\hline 02.05 .2010 & $\begin{array}{l}\text { Zusagen für } \\
\text { Griechenland }\end{array}$ & $\begin{array}{l}\text { Erhöhung auf } \\
80 \text { Mrd. Euro }\end{array}$ & & $\begin{array}{l}\text { Erhöhung auf } \\
30 \text { Mrd. Euro }\end{array}$ & \\
\hline 10.05 .2010 & Bereitstellung & $\begin{array}{l}440 \text { Mrd. Euro } \\
\text { (Garantien EFSF) }\end{array}$ & 60 Mrd. Euro & $\begin{array}{l}\text { Weitere } \\
250 \text { Mrd. Euro }\end{array}$ & $\begin{array}{l}<440 \text { Mrd. Euro } \\
\text { Darlehenskapa- } \\
\text { zität }\end{array}$ \\
\hline 28.11 .2010 & $\begin{array}{l}\text { Zusagen für } \\
\text { Irland }\end{array}$ & $\begin{array}{l}\text { Dänemark: } \\
\text { 0,4 Mrd. Euro } \\
\text { Schweden: } \\
\text { 0,6 Mrd. Euro } \\
\text { Großbritannien: } \\
\text { 3,8 Mrd. Euro }\end{array}$ & $\begin{array}{l}22,5 \mathrm{Mrd} . \\
\text { Euro }\end{array}$ & 22,5 Mrd. Euro & 17,7 Mrd. Euro \\
\hline 11.03 .2011 & Bereitstellung & $\begin{array}{l}\text { Erhöhung auf } \\
780 \text { Mrd. Euro } \\
\text { (Garantien EFSF) }\end{array}$ & & & $\begin{array}{l}440 \text { Mrd. Euro } \\
\text { Darlehens- } \\
\text { kapazität }\end{array}$ \\
\hline 25.03 .2011 & $\begin{array}{l}\text { Bereitstellung } \\
\text { für ESM ab } \\
2013^{3}\end{array}$ & $\begin{array}{l}80 \text { Mrd. Euro } \\
\text { (Grundkapital) } \\
620 \text { Mrd. Euro } \\
\text { (Garantien ESM) }\end{array}$ & & $\begin{array}{l}\text { Weiterhin } \\
250 \text { Mrd. Euro }\end{array}$ & $\begin{array}{l}500 \text { Mrd. Euro } \\
\text { Darlehens- } \\
\text { kapazität }\end{array}$ \\
\hline 17.05 .2011 & $\begin{array}{l}\text { Zusagen für } \\
\text { Portugal }\end{array}$ & & $26 \mathrm{Mrd}$. Euro & $26 \mathrm{Mrd}$. Euro & $26 \mathrm{Mrd}$. Euro \\
\hline 21.07 .2011 & $\begin{array}{l}\text { Zusagen für } \\
\text { Griechenland }\end{array}$ & & & \multicolumn{2}{|c|}{$\begin{array}{l}109 \text { Mrd. Euro (inklusive eines } \\
\text { freiwilligen Beitrags privater } \\
\text { Gläubiger in Höhe von } 37 \text { Mrd. } \\
\text { Euro) }\end{array}$} \\
\hline
\end{tabular}

55 Die Gipfelbeschlüsse enthalten folgende Formulierung: „Im Hinblick auf die Beteiligung des privaten Sektors werden wir strikt an den bewährten Grundsätzen und Verfahren des IWF festhalten“, Europäischer Rat: Erklärung der Staats- und Regierungschefs des Euro-Währungsgebiets, Brüssel, den 9. Dezember 2011, S. 6. Allerdings läuft dieser Verweis ins Leere, da es der IWF , [...] nach eigenen Angaben in seinen Kreditprogrammen noch nie zur Bedingung gemacht [hat], dass private Kreditgeber einer Regierung umschulden müssen“. Vgl. Frankfurter Allgemeine Zeitung: Einbezug privater Gläubiger in ESM unwahrscheinlich, 10.12.2011.

56 Werner Mussler: Auf Wiedervorlage, in: Frankfurter Allgemeine Zeitung, 10.12.2011. 


\begin{tabular}{|c|c|c|c|c|c|}
\hline Datum $^{1}$ & Adressat & Mitgliedstaaten $^{2}$ & $\begin{array}{c}\text { Europäische } \\
\text { Union }\end{array}$ & IWF & EFSF \\
\hline 26.10 .2011 & $\begin{array}{l}\text { Bereitstellung } \\
\text { über EFSF }\end{array}$ & $\begin{array}{l}\text { (die Garantien für } \\
\text { die EFSF bleiben } \\
\text { unverändert) }\end{array}$ & & & $\begin{array}{l}\text { Durch Hebe- } \\
\text { lung wird die } \\
\text { Wirkung der } \\
\text { restlichen Dar- } \\
\text { lehenskapazität } \\
\text { vervier- bis } \\
\text {-fünffacht }\end{array}$ \\
\hline 09.12 .2011 & $\begin{array}{l}\text { Bereitstellung } \\
\text { für IWF }\end{array}$ & $\begin{array}{l}\text { Eurostaaten und } \\
\text { andere Mitglied- } \\
\text { staaten prüfen, ob } \\
\text { sie dem IWF } \\
\text { weitere } 200 \text { Mrd. } \\
\text { Euro zur Verfü- } \\
\text { gung stellen }\end{array}$ & & $\begin{array}{l}\text { Ggf. Erhöhung } \\
\text { der Mittel des } \\
\text { IWF }\end{array}$ & \\
\hline \multicolumn{6}{|c|}{$\begin{array}{ll}1 & \text { Das genannte Datum bezieht sich jeweils auf das Datum der Absichtserklärung. } \\
2 & \text { Ist kein Staat genannt, so wurde die Zusage durch die Mitgliedstaaten der Eurozone getätigt. } \\
3 & \text { Die Mittel für den ESM werden nicht zusätzlich zur EFSF bereitgestellt, sondern der ESM wird die } \\
\text { EFSF schrittweise ablösen. }\end{array}$} \\
\hline
\end{tabular}

Quelle: Eigene Darstellung.

Angesichts der zahlreichen schwelenden Brandherde ist davon auszugehen, dass diese Krise die Europäische Union noch lange beschäftigen wird. Während die Staats- und Regierungschefs gemeinsam mit Zentralbanken und Finanzministern Löscharbeiten betreiben, die bei den sichtbaren Flammen, also den Symptomen der Krise ansetzen (siehe Tabelle 3), versuchen sie parallel einen Konsens über längerfristige und präventive Maßnahmen zu erreichen. Die jüngsten Gipfelbeschlüsse von Oktober und Dezember 2011 enthalten neue brandhemmende Details für das Gebäude der WWU. Diese institutionellen Neuerungen bedeuten eine Transformation des Regierens in der Wirtschaftssäule, die sich als Grundgerüst einer Europäischen Wirtschaftsregierung interpretieren lässt. Kritiker dieses Konzepts befürchten jedoch nach wie vor eine Spaltung des Binnenmarktes, einer Errungenschaft aller 27 EUMitgliedstaaten. Wie selbstständig die reformierte Eurozone von der Europäischen Union als ganzes operieren wird, ist daher unklar. Der jüngste Gipfel von Dezember 2011 hat den Präsidenten des Europäischen Rates gemeinsam mit den Präsidenten von Kommission und Eurogruppe beauftragt, im März 2012 einen Bericht zur weiteren Vertiefung der fiskalpolitischen Union vorzulegen, der unter anderem auf diese Frage eingehen soll. ${ }^{57}$ Auch wenn das Ergebnis des Umbaus der WWU offen ist, verdeutlicht die Chronologie der Beschlüsse auf höchster politischer Ebene und der dort verabschiedeten Maßnahmen, dass die Vertreter der Mitgliedstaaten erhebliche Veränderungen hin zu einer Europäischen Wirtschaftsregierung anstreben, deren Wirksamkeit, Reichweite und Folgen für die Europäische Union und für die Mitgliedstaaten selbst weiterer Untersuchung bedürfen.

57 Europäischer Rat: Erklärung der Staats- und Regierungschefs des Euro-Währungsgebiets, Brüssel, den 9. Dezember 2011, S. 4. 